\title{
A study of Bacterial profile and Antimicrobial susceptibility patterns of patients with ear infection in a tertiary care hospital in Northern India
}

\author{
Authors \\ Arshi $\mathbf{S}^{1}$, Bashir $\mathbf{H}^{2}$, Qadri $\mathrm{SS}^{3}$, Masoodi $\mathrm{T}^{4}$, Khurshid $\mathrm{S}^{5}$, Shafiq $\mathrm{A}^{6^{*}}$ \\ ${ }^{1}$ Associate Professor, Department of Microbiology, SKIMS Medical College \& Associated Hospital, \\ Bemina, Srinagar, J\&K \\ ${ }^{2}$ Demonstrator, Department of Microbiology, Government Medical College, Srinagar, J\&K \\ ${ }^{3}$ Assistant Professor, Department of Community Medicine, Government Medical College, Rajouri, J\&K \\ ${ }^{4}$ Lecturer, Department of Microbiology, SKIMS Medical College \& Associated Hospital, Bemina, Srinagar, \\ $\mathrm{J} \& \mathrm{~K}$ \\ ${ }^{5}$ Professor, Department of Microbiology, SKIMS Medical College \& Associated Hospital, Bemina, \\ Srinagar, J\&K \\ ${ }^{6}$ Associate Professor, Department of Surgery, Government Medical College, Srinagar, J\&K \\ *Corresponding Author \\ Dr Shafiq A \\ Associate Professor, Department of Surgery, Government Medical College, Srinagar, J\&K, India
}

\section{Introduction}

Otitis media (OM), an inflammation of the middle ear cleft, is a common problem worldwide. Globally, about 65-330 million people suffer from ear infection and $60 \%$ of them had significant hearing impairment. ${ }^{1}$ It is a prevalent problem for both children and adults especially in developing countries. Due to the low socio-economic status, overcrowding, poor hygiene, inadequate health care, and recurrent upper respiratory tract infection, the burden is high in low and middle income countries. ${ }^{2}$ Clinically, it is classified as acute, sub-acute and chronic depending on duration. Acute otitis media incidence rate is $10.85 \%$ while chronic suppurative otitis media incidence rate is $4.76 \%{ }^{3}$

Infection can be spread from middle-ear to vital structures such as mastoid, facial nerve, labyrinth, lateral sinus, meninges and brain leading to mastoid abscess, facial nerve paralysis, deafness, lateral sinus thrombosis, meningitis and intracranial abscess. ${ }^{4}$ Moreover normal flora of the skin such as Pseudomonas aeruginosa, Staphylococcus aureus, Proteus mirabilis, Klebsiella pneumonia and Escherichia coli that can easily enter through perforated ear have been reported as the main agents of otitis media. ${ }^{5}$

Empiric treatment of ear infection is not always appropriate since drug susceptibility patterns change overtime and empiric antibiotic therapy may not be effective at times and could contribute to development of antimicrobial resistance in the long run. ${ }^{6}$ Early, prompt and effective treatment of ear infection will significantly reduce both short and long term complications. Hence our study aims at determining the bacterial profile and 
antimicrobial susceptibility pattern of ear infections among patients in a tertiary care hospital in India.

\section{Material and Methods}

Study area and period: The study was conducted in the department of Microbiology of a tertiary care institute, Srinagar (Jammu \& Kashmir) over a period of three years i.e. from January 2015 to December 2018.

Study population: All thepatients attending the OPD with complaints of ear discharge during the period of three years were taken for the study purpose.

Culture and identification: After thorough clinical examination clinician collected discharge material with clean sterile swab stick. Whenever possible, two samples were collected. First sample of ear swab was for gram staining and second for culture. Each material was inoculated on to, bacterial blood agar, chocolate agar, and MacConkey agar. The MacConkey and blood agar plates were incubated in aerobic condition, whereas chocolate agar plate was kept in a candle jar, which was able to generate about 5-10\% $\mathrm{CO}_{2}$. After overnight incubation species isolated were identified by morphology, growth characteristics and bio-chemical reactions according to the standard conventional techniques.

Antimicrobial susceptibility testing: After identifying the isolate, their antibiotic sensitivity test was done on Mueller Hinton Agar by Kirby Bauer disc diffusion method according to CLSI recommendations. $^{7}$

Inclusion criteria: Patients from age group of 0 to 70 years and of either sex, who complained of ear discharge, were included in the study. Informed consent was taken from all patients before recruiting in the study.

Data analysis: Data was entered in Microsoft excel office and the results were expressed in the form of tables, bars and proportions.

\section{Results}

The study was conducted in the Department of Microbiology of a tertiary care institute, Srinagar and Kashmir. The sample ie ear discharge received from patients attending the OPD from January 2015 to December 2018 was processed for isolation and identification of bacterial pathogens according to the standard microbiological techniques.

A total of 145 samples were obtained for a period of 3 years comprising of $85(58.6 \%)$ females and $60(41.3 \%)$ males. Most common age group involved was $20-40 \mathrm{yrs}(55.1 \%)$ followed by $0-20$ yrs $(24.1 \%)$ whereas less number of isolates were found in elderly age groups i.e. 40-60yrs (10.1\%) and $>60$ yrs (16.3\%) respectively.(Table-1).

The most predominant bacterial isolates was Staphylococcus aureus 60 (40\%) followed by Pseudomonas spp 40 (26.6\%), Coagulase negative Staphylococcus 20 (13.33\%), Enterococcus spp. 10 $(6.66 \%)$, whereas less number of isolates 5 each (3.3\%) were from Klebisella, E. coli, Moraxella and candida respectively (Table-2, Figure-1).

A single growth of organism was found in $115(79.3 \%)$, mixed growth was observed in $20(13.9 \%)$ cases, however no growth was seen in $10(6.89 \%)$ (Figure-2).

Occurrence of gram positive organisms was higher than gram negative cases. With gram positive isolation rate was $90(60 \%)$ and for gram negative organisms it was 55(36.66\%).

Antibiotic sensitivity and resistant pattern of Staphylococcus aureus shows that 80 Staphylococcus spp 20 (13.33\%) were CoNS. Among coagulase positive Staphylococcus 15(25\%) were MRSA. $100 \%$ sensitivity was observed for Vancomycin, Teicoplanin and Linezolid.83.33\% resistance was seen with Ciprofloxacin,75\% with Cotrimoxazole, $66.66 \%$ with Erythromycin. $41.66 \%$ with Clindamycin, $75 \%$ with Gentamicin, $33.33 \%$ with Azithromycin (Figure-3).

Pseudomonas showed $100 \%$ sensitivity to Colistin and Polymyxin B. Susceptibility pattern showed $62.5 \%$ sensitivity with Amikacin, $75 \%$ with 
Ciprofloxacin. Most commonly used drug against Pseudomonasie Ceftazidime showed sensitivity of 45\% (Figure-4).

Coagulase negative Staphylococcus isolates showed $100 \%$ sensitivity to Vancomycin, Linezolid, $30 \%$ to TrimethoprimSulfamethoxazole and $70 \%$ to Oxacillin (Figure5).

Among Enterococcal isolates $100 \%$ sensitivity was seen for Vancomycin Linezolid and Erythromycin. However all the isolates were 100\% resistant to Ampicillin and Ciprofloxacin (Figure-6).

With regard to Klebisella spp isolate, $44 \%$ were sensitive to Ceftriaxone and $77.8 \%$ sensitive to Trimethoprim-Sulfamethoxazole, $16.7 \%$ were sensitive to Ciprofloxacin and $38.9 \%$ sensitive to Gentamycin. It showed high frequency of resistance to Ampicillin. Among other gram negative bacteria like E.coli $66 \%, 16.7 \%$ and $11 \%$ of the isolates were sensitive to Ceftriaxone,
Gentamycin and Ciprofloxacin respectively (Table-3).

Table 1: Age and gender distribution of patients $(\mathrm{n}=145)$

\begin{tabular}{|l|c|c|c|}
\hline Characteristics & No. & \% \\
\hline \multirow{4}{*}{ Age groups } & $0-20$ & 35 & 24.1 \\
\cline { 2 - 4 } & $20-40$ & 80 & 55.1 \\
\cline { 2 - 4 } & $40-60$ & 15 & 10.1 \\
\cline { 2 - 4 } & $>60$ & 15 & 16.3 \\
\hline \multirow{2}{*}{ Gender } & & & \\
\cline { 2 - 4 } & Male & 60 & 41.3 \\
\cline { 2 - 4 } & Female & 85 & 58.6 \\
\hline
\end{tabular}

Table 2: Bacterial isolates of the ear discharge $(n=145)$

\begin{tabular}{|l|c|c|}
\hline Bacterial Isolates & No. & \% \\
\hline Staphylococcus aureus & 60 & 40 \\
\hline Pseudomonas & 40 & 26.6 \\
\hline Coagulase negative & 20 & 13.3 \\
\hline Enterococcus & 10 & 6.6 \\
\hline Klebisella & 5 & 3.3 \\
\hline E.coli & 5 & 3.3 \\
\hline Morxella & 5 & 3.3 \\
\hline Candida & 5 & 3.3 \\
\hline
\end{tabular}

Figure 1: Bacterial isolates of the ear discharge $(n=145)$

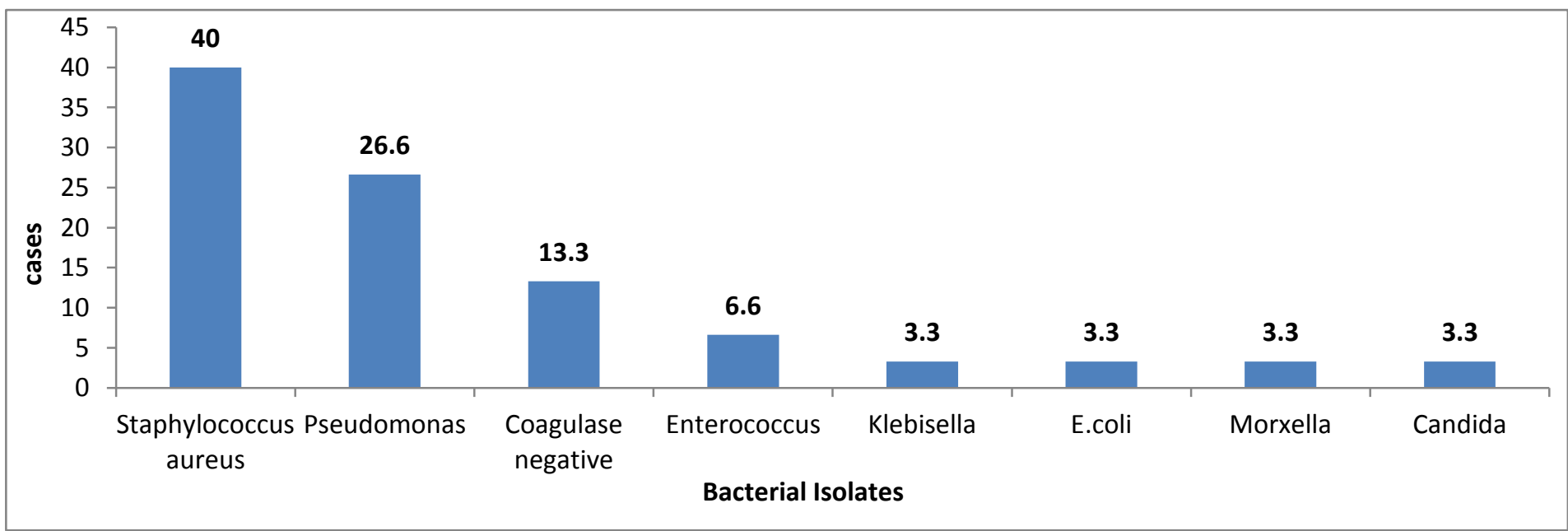

Figure 2: Type of growth seen in ear discharge of cases $(n=145)$

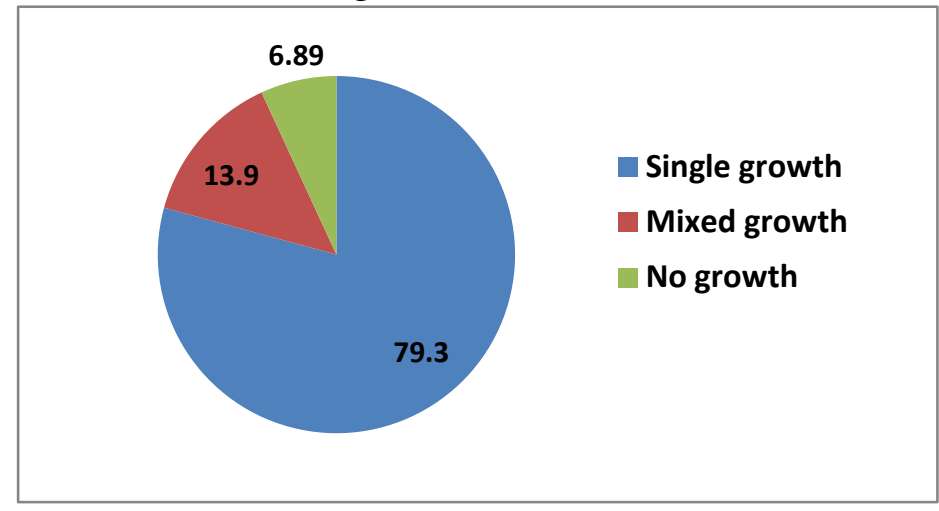




\section{JMSCR Vol||07||Issue||04||Page 66-72||April}

Figure 3: Antibiotic Sensitivity and Resistant pattern of Staphylococcus aureus $(n=60)$

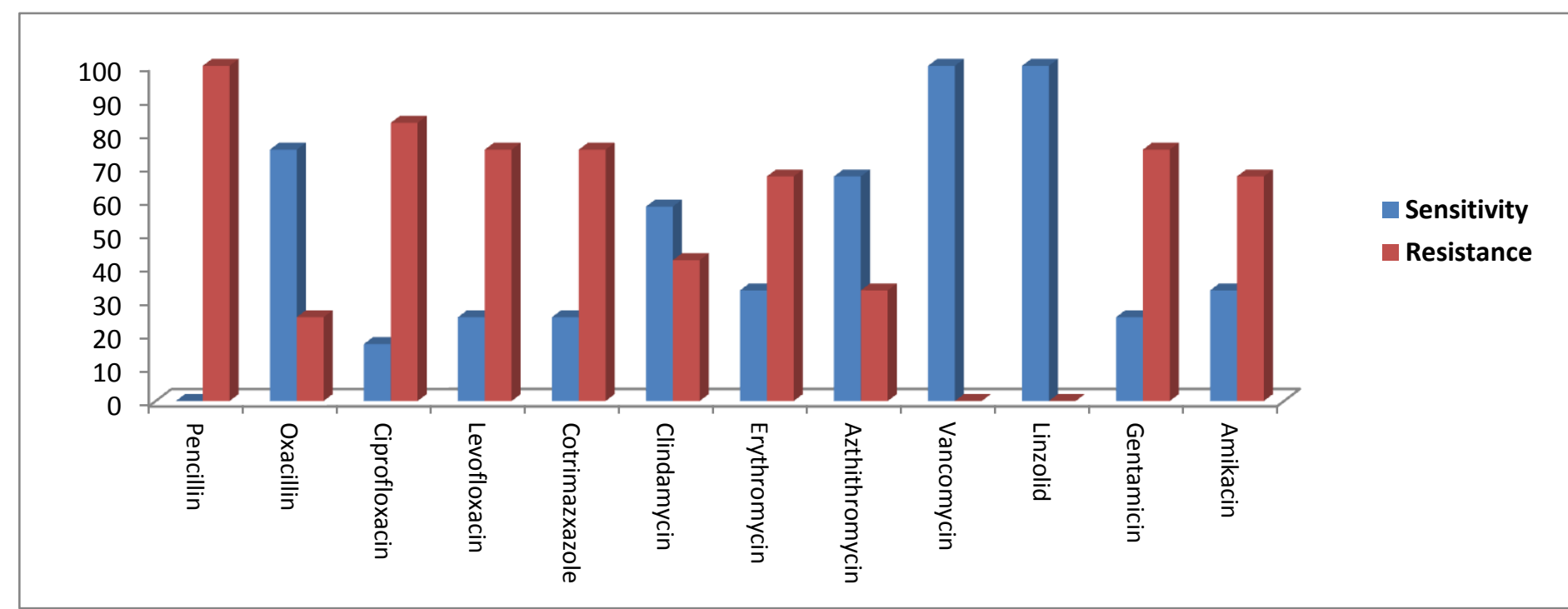

Figure 4: Antibiotic Sensitivity and Resistant pattern of Pseudomonas $(\mathrm{n}=40)$

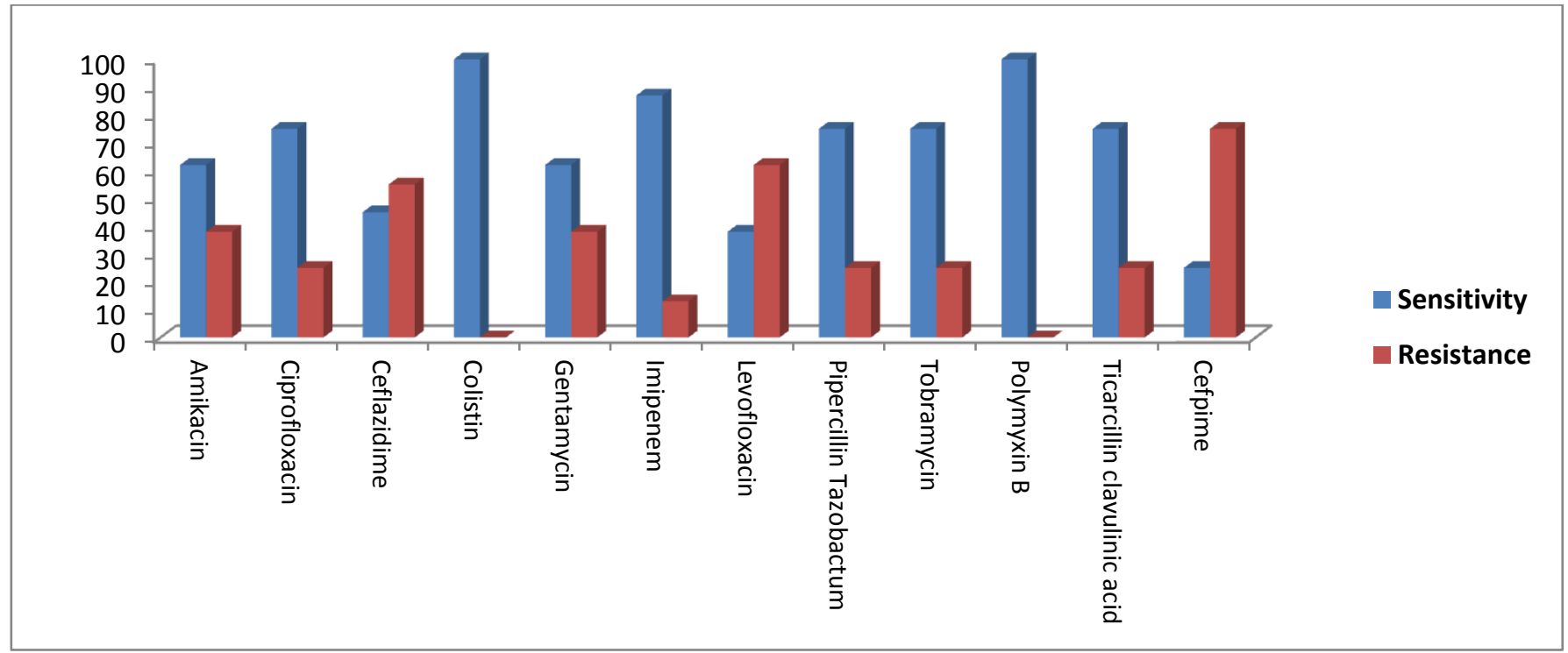

Figure 5: Antibiotic Sensitivity and Resistant pattern of Coagulase negative $(\mathrm{n}=20)$

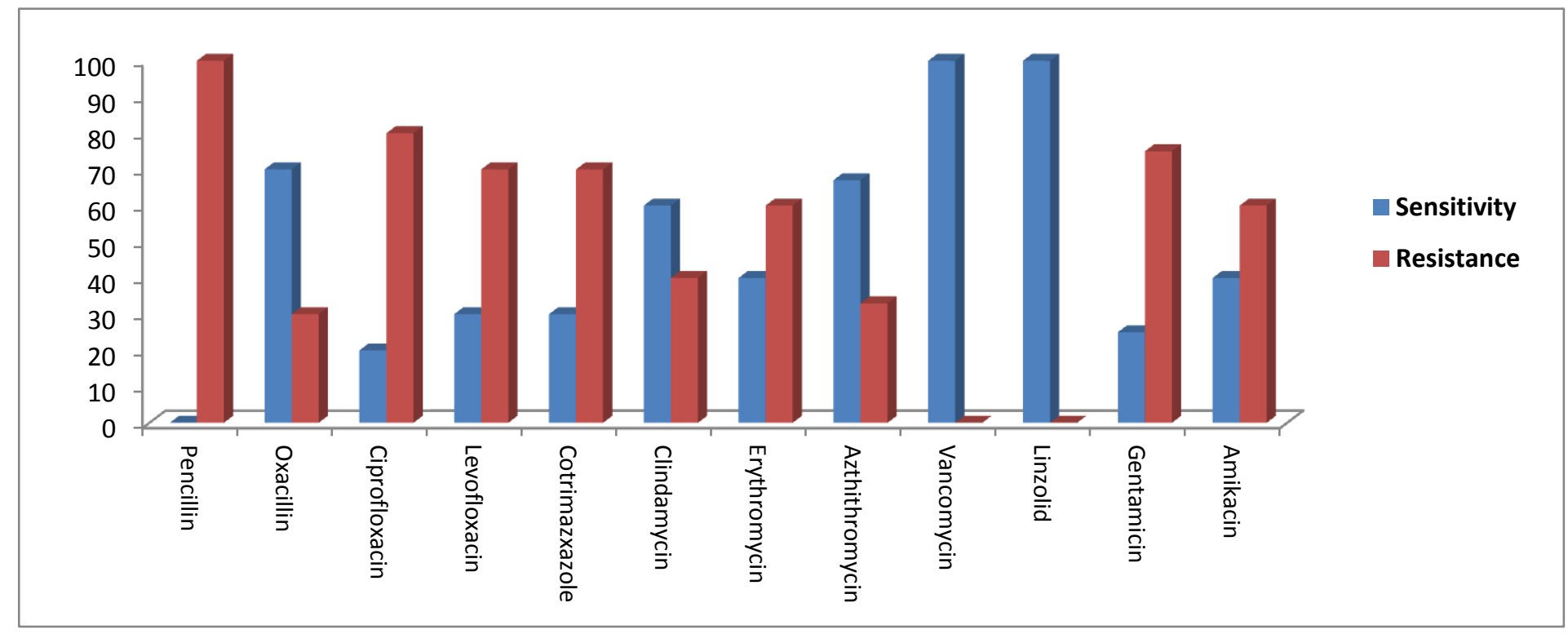


Figure 6: Antibiotic Sensitivity and Resistant pattern of Enterococcus $(\mathrm{n}=10)$

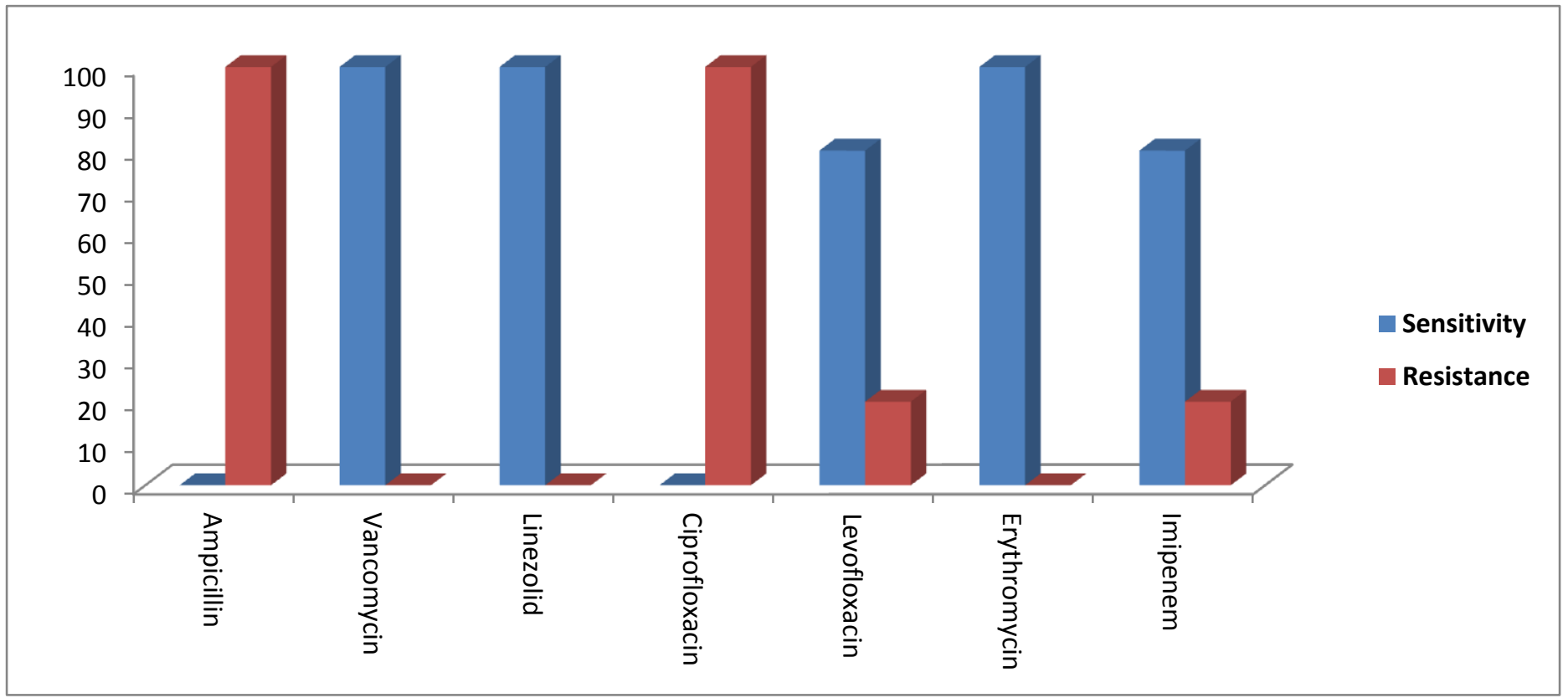

Table 3: Antimicrobial sensitivity pattern of gram negative bacilli

\begin{tabular}{|l|c|c|}
\hline Drugs & E.coli & Klebisella \\
\hline Ceftriaxone & 66 & 44 \\
\hline Ampicillin salbactam & 22.2 & 25 \\
\hline Ciprofloxacin & 11 & 16.6 \\
\hline Levofloxacin & 33.3 & 12.5 \\
\hline Tetracycline & 50 & 8.9 \\
\hline Cotrimaxazole & 66.7 & 77.8 \\
\hline Gentamycin & 16.7 & 38.9 \\
\hline Amikacin & 11.1 & 12.5 \\
\hline Imipenem & 90 & 89 \\
\hline Ampicillin & 88.9 & 100 \\
\hline
\end{tabular}

\section{Discussion}

Otitis media is commonest disease to come across in day to day practice. Although, a non- fatal disease which could lead to hearing loss, facial paralysis and many neurological complications. The reason being low socio economic status of people with poor affordability, neglecting the discharging ear which leads to inadequate and improper treatment. Hence, early diagnosis and effective treatment is necessary to avoid complications. Antibiotics are usually started on the basis of its efficacy, resistance pattern, cost and side effects. Therefore, it is very important to know its antibiotic sensitivity for effective treatment.

In our study, a total of 145 samples were obtained comprising of $85(58.6 \%)$ females and $60(41.3 \%)$ males. Most common age group involved was 2040 yrs $(55.1 \%)$ followed by $0-20$ yrs $(24.1 \%)$. Similar results were found in a study by Panchal $\mathrm{PD}$ et $\mathrm{al}^{3}$, Akter $\mathrm{S}$ et $\mathrm{al}^{\mathbf{8}}$ who in their respective studies found that common age group involved was 21-30 years with female predominance $(62 \%$ $\& 64.35 \%)$ respectively. However in a study by Wasihun $\mathrm{AG}$ et $\mathrm{al}^{9}$ it was seen that the paediatric age group (6-10 yrs) was most commonly involved with male predominance $(64.8 \%)$.

Our study revealed that the most predominant bacterial isolates were Staphylococcus aureus (40\%) followed by pseudomonas (26.6\%) and coagulase negative $(13.33 \%)$. Similar results were observed in a study by Agarwal $\mathrm{A}$ et $\mathrm{al}^{\mathbf{1 0}}$, Wasihun AG et $\mathrm{al}^{\mathbf{9}}$ and Akter $\mathrm{S}$ et $\mathrm{al}^{\mathbf{8}}$ who in their studies found that Staphylococcus spp was the most predominant organism $(37.6 \%, 28.4 \%$ \& $24.42 \%$ ), followed by Pseudomonas aeruginosa $(32.8 \%, 16.7 \%$ \& $23.26 \%)$ respectively. However in a study Panchal PD et $\mathrm{al}^{3}$, it was found that the most common isolate was Pseudomonas aeruginosa (25.88\%) followed by Staphylococcus aureus $(21.17 \%)$. 
Our study revealed that single growth of organism was found in $79.3 \%$ followed by mixed growth in $13.9 \%$ cases. Similar results were found in a study by Agarwal $A$ et $\mathrm{al}^{10}$ who also found monomicrobial bacterial isolate was $80 \%$ of cases while $8 \%$ had mixed growth. In a study by Panchal PD et al $^{3}$ majority $(96.6 \%)$ samples had single organism isolated on culture study, while (3.40\%) had mixed growth. In studies by Akter S etal $^{8}$ and Wasihun AG et $\mathrm{al}^{9}$, it was found that $81.40 \%$ and $60.5 \%$ had single bacterial infection while $18.60 \%$ and $39.5 \%$ had mixed bacterial infections respectively.

While looking for Antibiotic sensitivity Staphylococcus spp. showed sensitivity of $16.66 \%$ with Ciprofloxacin. In some of the studies sensitivity was higher (83-95\%). Vancomycin, linezolid, Teicoplanin showed $100 \%$ sensitivity thus making these agents drug of choice. Pseudomonas showed higher sensitivity to Imipenem, Colistin, Polymyxin B and PipercillinTazobactum. Sensitivity with Amikacin (62.5\%) was in concordance with study by Madana $\mathrm{J}$ et $\mathrm{al}^{11}$ and Tahir $\mathrm{M}$ et $\mathrm{al}^{12}$. Sensitivity with quinolones was 75 and $37.5 \%$ respectively for Ciprofloxacin and Levofloxacin. These observations are in contrast to the other studies showing higher sensitivity of $90-92 \% .{ }^{13}$ The declining sensitivity trend with quinolones may be due to a number of factors including injudicious use, inappropriate dosage, and easy accessibility.

\section{Conclusion and Recommendations}

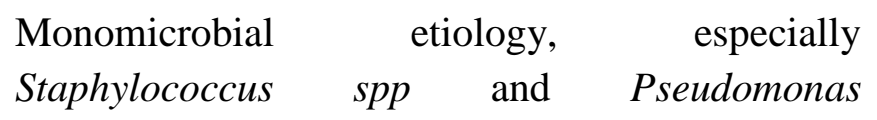
aeruginosa, was found to be the most common in our study. Staphylococcus species are highly resistant to ampicillin and ciprofloxacin. Pseudomonas aeruginosais becoming less sensitive against commonly used antimicrobials, viz. ciprofloxacin, ofloxacin, cephalosporins, and gentamicin. Therefore, evaluation of microbiological pattern and their antibiotic sensitivity pattern is becoming helpful in prescribing empirical antibiotics for successful treatment, thus minimizing the risk of complications and emergence of resistant strains. Hence we recommend antimicrobial surveillance to make the right recommendation of antibiotics along with strict adherence to antibiotic use policy to reduce the spread of drug-resistant microbes and associated complications. Further researches are needed to identify high resistance strains using molecular techniques.

Source of support:-Nil

Conflict of interest:-None declared.

\section{References}

1. Woodfield $G$ and DugdaleA. Evidence behind the WHO guidelines: hospital care for children: What is the most effective antibiotic regime for chronicsuppurative otitis media in children. $\mathbf{J}$ Tropical Pediatric 2008; 54(3):151-156.

2. Kumar H, Seth S. Bacterial and fungal study of 100 cases of chronic suppurative otitis media. J ClinDiagn Res 2011; 5:1224-1227.

3. Panchal PD, Patel BV. Evaluation of bacteriological profile and antibiotic susceptibility pattern of patients with otorrhea in a tertiary care teaching hospital. Int J Res Med Sci 2015; 3:316770.

4. Ulug M and Oksay B. Bacterial isolates and antibiotic susceptibility patterns of ear discharge from patients with chronic suppurative otitis media and review of the literature. June 2014 Sylwan 158(6):1-25.

5. Abera B and Kibret M. Bacteriology and antimicrobial susceptibility of otitis media at dessie regional health research laboratory, Ethiopia. Ethiopian J Health Develop 2011; 25(2):161-167.

6. Grevers G. Challenges in reducing the burden of otitis media disease: an ENT perspective on improving management and prospects for prevention. Int $\mathrm{J}$ Pediatr Otorhinolaryngol. 2010; 74(6):572-7. 
7. Mackie, McCartney. Practical medical microbiology. In: Mackie, McCartney, eds. 14th ed.US: Kundli Press, Elsevier Publishers; 2012.

8. Akter S, Shamsuzzaman SM, Nehar N, Siddiqui I, JahanF,Islam S. Bacterial isolates and drug susceptibility patterns of ear discharge from patients with ear infection at ShaheedMonsur Ali Medical College. Bangladesh J Med Microbiol 2015; 9 (2): 20-23.

9. Wasihun AG and Zemene Y. Bacterial profile and antimicrobial susceptibility patterns of otitis media in AyderTeaching and Referral Hospital, Mekelle University, Northern Ethiopia. SpringerPlus 2015; 4:701.

10. Agrawal A, Kumar D, Goyal A, Goyal S, Singh N, Khandelwal G. Microbiological profile and their antimicrobial sensitivity pattern in patients of otitis media with ear discharge. Indian J Otol 2013; 19:5-8.

11. Madana J, Yolmo D, Kalaiarasi R, Gopalakrishnan S, Sujata S. Microbiological profile with antibiotic sensitivity pattern of cholesteatomatous chronicsuppurative otitis media among children. Int $\mathrm{J}$ Pediatr Otorhinolaryngol 2011;75:1104-8.

12. Tahir M, Jawaid A, Abdullah A, Najam MA. Bacterial culture and sensitivity in active chronic otitis media: 500 cases in combined military hospital Rawalpindi. Pak J Otolaryngol 2012;28:56-8.

13. Gorems K,Beyene G,Berhane M, Mekonnen Z. Antimicrobial susceptibility patterns of bacteria isolated from patients with eardischarge in Jimma Town, Southwest, Ethiopia. BMC Ear, Nose and Throat Disorders 2018; 18:17. 